\title{
Are lasers as effective as scaling for chronic periodontitis?
}

\section{Abstracted from}

Sgolastra F, Petrucci A, Gatto R, Monaco A.

Efficacy of Er:YAG laser in the treatment of chronic periodontitis: systematic review and meta-analysis. Lasers Med Sci 2011 May 7. [Epub ahead of print]

Address for correspondence: F Sgolastra, Department of Health Sciences, University of L'Aquila,

San Salvatore 1, Building Delta 6, 67100 L'Aquila, Italy.E-mail: fabrizio.sgolastra@gmail.com

\section{Question: In patients with chronic periodontitis is treatment with erbium-doped:yttrium- aluminiumgarnet (Er:YAG) laser as effective as scaling and root planing (SRP)?}

Data sources Medline, Cochrane Database of Systematic Reviews (CDSR), Database of Abstracts of Reviews of Effects (DARE), CINAHL, Science Direct, ISI Web of Science, and SCOPUS. The following journals were hand searched: Journal of Periodontology, International Journal of Periodontics and Restorative Dentistry, Journal of Clinical Periodontology, Journal of Dental Research, Journal of Periodontal Research, Periodontology 2000, Journal of Dentistry, Journal of American Dental Associations, Journal of Clinical Dentistry, Lasers in Medical Science, Lasers in Surgery and Medicine, Clinical Oral Investigations, and Photomedicine and Laser Surgery as well as the reference lists of included articles.

Study selection Randomised controlled clinical trials (RCTs) comparing Er:YAG laser with manual or ultrasonic SRP alone were included. No language restrictions were applied.

Data extraction and synthesis Data were collected by two independent reviewers with only numeric data being extracted. Data were combined for meta-analysis with the effect size being estimated and reported as the mean difference (MD) for continuous variables using a random effects model. Heterogeneity was assessed using the $\div$ 2-based Q-statistic method and $\mathrm{I}^{2}$ measurement.

Results Five trials met the inclusion criteria. Five trials (85 patients and 3,564 sites) were entered in the meta-analysis to investigate clinical attachment level (CAL) gain, pocket depth (PD) reduction and gingival recession (GR). All studies reported significant intragroup improvement in clinical and microbiological parameters in patients treated with the Er:YAG laser. However, three studies did not report a significant difference between Er:YAG laser and SRP groups in CAL gain, PD reduction or GR changes. The meta-analysis revealed no significant differences for any investigated parameters at six and 12 months. Conclusions Significant heterogeneity, a high risk of bias in three of the five included studies, and methodological shortcomings indicate that the results should be considered with caution. Future long-term, well-designed RCTs are needed to assess the scientific evidence of Er:YAG laser efficacy as an alternative treatment strategy to SRP.

\section{Commentary}

This well done systematic review parallels the findings of four previous systematic reviews on the same topic, and comes to similar conclusions. ${ }^{1-4}$ There is:

- No apparent difference in clinical efficacy between scaling and root planing and Er:YAG laser therapy

- Significant risk of bias in the reported trials

- A need for larger scale, masked randomised controlled trials

The current review improves on previous reviews in several important aspects. It:

- Improves the previous search strategies and updates the literature searches

- Identifies and includes two new studies

- Performs a quality assessment, meta-analysis, and includes an examination of heterogeneity and publication bias

The systematic review itself follows current best practices, including adherence to the QUORUM statement and the Cochrane Collaboration recommendations. Further, quality assessment of the individual trials followed the CONSORT guidelines. The consistent results and recommendations of five systematic reviews over a 7-year period raises the following question: what are the critical elements that have so far been missed in clinical trials? In answering this question two elements regarding laser therapy for treating periodontal disease are relevant. First, the clinical presentation of the disease is localised to particular teeth and sites. Second, the argument for lasers is that they can access deep sites, furcations, grooves, concavities and deep pockets better than scalers.

From this review it is clear that the missing critical element of all clinical trials is the inclusion of only those sites where one might expect lasers to excel (eg $>6 \mathrm{~mm}$ pockets or furcations with calculus). Instead, as indicated in the current review, the reported clinical trials include sites with modest pocket depth (eg patients with sites $\geq 4-5$ $\mathrm{mm}$ ). The selection of shallow(er) sites narrows the dynamic range of the outcomes. With a narrow dynamic range of outcomes (eg $4 \mathrm{~mm}$ to $3 \mathrm{~mm}$ ), there is a substantial possibility that there would be no difference between scaling/root planing and lasers. The statistical result would show no significant difference, even if there was one. In other words, with a narrow dynamic range, there is a high likelihood of a false negative outcome. To address this issue trials might specifically exclude sites with modest depth.

Another issue is the use of single or multiple sites might in a mouth. In the current review (Table 4), the handling of data 
in the five reported studies is not clear. Table 4 indicates 19-26 patients in the trials, with $\sim 70-700$ teeth, and $~ 70-1,600$ sites. One wonders whether sites were averaged in a standard fashion across all studies. Current thinking favours the subject (or the tooth) as the unit of observation. Therefore, if multiple sites are reported for a tooth, they should be averaged for that tooth. Similarly, if multiple teeth are included in a subject, these should be averaged for the subject.

The third item is power calculations. Given that five clinical trials indentified the same issues: few patients in the identified trials $(\mathrm{n}=19-26)$; minimal differences in the clinical attachment change following therapy $(\sim 0.2-2.0 \mathrm{~mm})$; and a wide standard deviation of this change $(\sim 0.7-2.0 \mathrm{~mm})$, one would expect clinical trialists to use this information to carry out and report power calculations. This is critical for the trial itself (to insure the time, effort and expense are optimally invested), and as an ethical issue. Informed consent mandates involvement of patients in research only when there is a possibility to improve care. The absence of appropriate power calculations violates this ethical responsibility. There are also the issues of masking. Hopefully, it is now clear from the multitude of reporting guidelines (eg http://www.equator-network.org/), that masking is a critical reporting element to reduce bias.

Finally, given the five systematic reviews, carried out over seven years, all with equivocal findings, one cannot help but ask why manufacturers expect clinicians to pay a premium for lasers, when manufacturers are unwilling to invest in high quality clinical trials?

Richard Niederman

Centre for Evidence-Based Dentistry, The Forsyth Institute, Boston, Massachusetts, USA.

1. Ishikawa I, Aoki A, Takasaki AA. Potential applications of Erbium:YAG laser in periodontics. J Periodontal Res 2004; 39: 275-285.

2. Chanthaboury R, Irinakis T. The use of lasers for periodontal debridement: marketing tool or proven therapy? J Can Dent Assoc 2005; 71: 653-658.

3. Cobb CM. Lasers in periodontics: a review of the literature. J Periodontol 2006; 77: 545-564.

4. Schwarz F, Aoki A, Becker J, Sculean A. Laser application in non-surgical periodontal therapy: a systematic review. / Clin Periodontol 2008; 35: 29-44.

Evidence-Based Dentistry (2011) 12, 80-81. doi:10.1038/sj.ebd.6400811 